Research Paper

\title{
Value of pretreatment 18F-FDG PET/CT in prognosis and the reflection of tumor burden: a study in pediatric patients with newly diagnosed neuroblastoma
}

\author{
Shuai Man, MD#; Jie Yan, MD#; Jie Li, MD, PhD ${ }^{1}$; Yanna Cao, MD, PhD ${ }^{1}$; Jiajian Hu, MD, PhD; Wenchao \\ Ma, MD, PhD2; Jianjing Liu, MD, PhD ${ }^{\circledR}$; Qiang Zhao, MD, PhD ${ }^{\bowtie}$ \\ 1. Department of Pediatric Oncology, Tianjin Medical University Cancer Institute and Hospital, National Clinical Research Center for Cancer, Key Laboratory \\ of Cancer Prevention and Therapy of Tianjin, Tianjin's Clinical Research Center for Cancer, Tianjin, China. \\ 2. Department of Molecular Imaging and Nuclear Medicine, National Clinical Research Center for Cancer, Tianjin Medical University Cancer Institute and \\ Hospital, Tianjin, Tianjin's Clinical Research Center for Cancer, Tianjin, China.
}

\#These authors have contributed equally to this work.

$\triangle$ Corresponding authors: Qiang Zhao, MD, PhD, Professor, E-mail: zhaoqiang@tjmuch.com Tel.: 022-23359984, Fax:022-23359984; Jianjing Liu, MD, PhD, E-mail: lijbhm@163.com, Tel.: 86-13920690301, Fax: 022-23340123.

(c) The author(s). This is an open access article distributed under the terms of the Creative Commons Attribution License (https://creativecommons.org/licenses/by/4.0/). See http://ivyspring.com/terms for full terms and conditions.

Received: 2021.01.15; Accepted: 2021.02.11; Published: 2021.02.24

\begin{abstract}
Fluorine-18 fluorodeoxyglucose (18F-FDG) PET/CT has been commonly used in pediatric patients with newly diagnosed neuroblastoma (NB) for diagnosis. We retrospectively reviewed 40 pediatric patients with newly diagnosed NB who underwent 18F-FDG PET/CT. Clinicopathological factors and metabolic parameters including maximum standardized uptake value (SUVmax), metabolic tumor volume (MTV), and total lesion glycolysis (TLG) on PET/CT were evaluated as predictive factors for progression-free survival (PFS) and overall survival (OS) by univariate and multivariate analysis. Spearman rank correlation analyses were used to estimate the correlations between clinical factors and PET findings. The mean follow-up after 18F-FDG-PET/CT was 32.9 months. During the follow-up period 15 (37.5\%) patients experienced progression, and $9(22.5 \%)$ died. MTV $(P=0.001)$ and TLG $(\mathrm{P}=0.004)$ remained significant predictive factors for tumor progression, along with lactate dehydrogenase $(\mathrm{LDH})$, neuron-specific enolase (NSE) and bone metastasis. Univariate analysis showed that bone metastasis, LDH (>1064 IU/L), NSE (>364.4 ug/L), MTV $(>191 \mathrm{~cm} 3)$ and TLG (>341.41 g) correlated with PFS, and LDH (>1064 IU/L), NSE $(>364.4 \mathrm{ug} / \mathrm{L})$ and MTV $\left(>191 \mathrm{~cm}^{3}\right)$ correlated with OS $(p<0.05)$. In multivariate analysis, MTV and bone metastasis were independent prognostic factors for PFS ( $p=0.001$ and 0.023 , respectively), and MTV remained the only independent prognostic factor for OS $(p=0.004)$. We also found that there were correlations between semiquantitative PET/CT parameters and clinical features in NB. Our results suggested that $18 \mathrm{~F}-\mathrm{FDG}$ PET/CT was a useful tool to predictive progression and to reflect tumor burden for patients with NB.
\end{abstract}

Key words: 18F-FDG PET/CT; MTV; TLG; neuroblastoma; progression; tumor burden

\section{Introduction}

Neuroblastoma (NB) is the most common malignant extracranial solid tumor in paediatric patients that occurs anywhere in the sympathetic nervous system, but mostly occurs in the adrenal medulla, and accounts for $7 \%-10 \%$ of all paediatric tumors $[1,2]$. Approximately $60 \%$ of patients with NB have metastatic disease at presentation. Common metastasis sites include the bone marrow, bone, lymph nodes, and liver [2]. More than $50 \%$ of patients with NB are diagnosed as high risk with a long-term survival probability $<50 \%$ [3]. The factors related to prognosis include the age at diagnosis, tumor histology type, tumor biological characteristics, primary tumor site, disease stage, and tumor response to treatment. Based on the risk classification of the Children's Oncology Group (COG), patients were 
classified into low-risk, intermediate-risk and high-risk groups with different outcomes and different treatment needs [4].

2-deoxy-2-[18F] fluoro-D-glucose (18F-FDG) positron emission tomography/computed tomography (PET/CT) is a non-invasive, whole-body imaging examination that provides complete anatomical information with $\mathrm{CT}$ and detects the extent of FDG uptake in the primary tumor and metastases [5, 6]. Previous studies have demonstrated the important role of 18F-FDG PET/CT in staging disease, detecting recurrence, and evaluating treatment response for renal cell carcinoma [7], non-small cell lung cancer [8], breast cancer [9], etc. Tumor cells have a higher metabolic activity than normal cells and usually show higher FDG uptake. The most commonly used PET/CT parameter is the maximum standardized uptake values (SUVmax). Some recent studies have shown that metabolic tumor volume (MTV) and total lesion glycolysis (TLG) can provide important information about metabolism and prognosis for various human tumors. There are a few studies on the application of 18F-FDG PET/CT for NB $[2,10,11]$. However, most of these studies focused on comparing the diagnostic efficacy of 18F-FDG PET/CT and other imaging methods, such as iodine 123 metaiodobenzylguanidine (I-123 MIBG) [11, 12], or only evaluated the diagnostic value of 18F-FDG PET/CT for NB $[13,14]$. To the best of our knowledge, there are few studies that have assessed the prognostic significance of the metabolic parameters on PET/CT or have evaluated the correlation between semiquantitative PET/CT parameters and clinical features in patients with NB $[15,16]$. Therefore, we conducted a retrospective analysis to determine whether 18F-FDG PET/CT can provide prognostic information and reflect tumor burden in NB.

\section{Materials and methods}

\section{Patients}

We retrospectively reviewed the records of all paediatric patients (age $<18$ years) with NB who had undergone 18F-FDG PET/CT before treatment from January 2013 to December 2018. The inclusion criteria were as follows: 1) diagnosed by ultrasonographyguided fine-needle aspiration or biopsy and final postoperation pathology and 2) received comprehensive treatment after 18F-FDG PET/CT imaging. The exclusion criteria were as follows: 1 ) any treatment procedures before 18F-FDG PET/CT investigation and 2) patients without a pathological diagnosis. The data obtained from the clinical medical records included age, sex, risk stratification, MYCN amplification, laboratory test parameters including lactate dehydrogenase (LDH), neuron-specific enolase (NSE), and follow-up information. Next, correlations between initial TLG or SUVmax values and serum markers were investigated in all patients. All patients were followed up for more than 6 months (mean, 32.9 months; range, 6-77 months). Patients were classified to progression group and the non-progression group. Local tumor progression, tumor recurrence, metastasis and death due to the disease were considered as progression. Recurrence referred to the complete disappearance of the tumor 1 month later with local or metastatic tumor lesions that appeared again. Metastatic lesions found on any image throughout the study period were recorded. BMI was verified by bone marrow biopsy (BMB). Metastatic lesions found on any image, such as $\mathrm{CT}$, magnetic resonance imaging (MRI) and bone scan, throughout the study period were recorded.

This study protocol was approved by the institutional review board of Tianjin Medical University Cancer Institute and Hospital. All methods were carried out in accordance with the relevant guidelines and regulations.

\section{F-FDG PET/CT}

The NB patients fasted for at least 6 hours prior to the examination and had a blood glucose lower than $10 \mathrm{mmol} / \mathrm{L}$. The intravenous injection of FDG ranged from $4.44-5.55 \mathrm{MBq} / \mathrm{kg}$, depending on the body surface area. Twenty-three patients were given oral or i.v. sedation for PET scans. PET/CT examinations were performed 60 minutes after injection of the tracer using a GE PET/CT system (Discovery 690). The examinations included a head-to-toe CT scan $(80 \mathrm{kV} ; 50-100 \mathrm{mAs}$.) and a 3-dimensional (3D) PET scan (2 minutes per bed, 6-7 beds). The rotation time was 0.6 . The slice thickness was $3.75 \mathrm{~mm}$. The increment was 3.27 . The pitch was 0.984. The images were displayed on the Xeleris workstation.

\section{Image analysis}

Two experienced nuclear medicine physicians reviewed the 18F-FDG PET/CT images independently. Depending on the axis and the coronal and sagittal projections, the physicians placed the volume of interest (VOI) on the primary tumor. The metabolic parameters, such as SUVmax, SUVmean and MTV, were measured on PET/CT images using volume viewer software, and TLG was then calculated as [TLG $=\mathrm{SUVmean} \times \mathrm{MTV}]$. In our study, we selected an SUVmax of $42 \%$ as the threshold for generating the VOI. 


\section{Statistical analysis}

Statistical analyses were performed using SPSS software (version 25.0 for Windows; SPSS INC.). Continuous data are described as the mean \pm standard deviation (mean $\pm \mathrm{SD}$ ) or median and range, depending on whether they followed a normal distribution, and categorical variables are described as numbers.

- PET/CT parameters and clinicopathological factors were compared between the progression group and the non-progression group using independent t-tests, Mann-Whitney $U$ tests, $\chi 2$ tests, and Fisher exact tests.

- Spearman rank correlation analyses were used for to analyse the relationship between serum biomarker levels (LDH, NSE) and semiquantitative PET/CT parameters (SUVmax, MTV, TLG). The relationships among MYCN amplification, risk stratification, lymph node metastasis, and PET/CT parameters were analysed with t-tests or Mann-Whitney U tests.

- Progression-free survival (PFS) and overall survival (OS) were calculated to evaluate the risk of progression and death. PFS was defined as the time from the day of diagnosis to the first documented evidence of disease progression or recurrence, based on any radiographic examination. OS was defined as the time from the day of diagnosis to the date of death or last follow-up. Survival curves were estimated using the Kaplan-Meier method to calculate cumulative PFS rates and OS rates and compared with the log-rank test.

- Receiver operating characteristic (ROC) curve analysis was used to find the best cut-off value to classify LDH, NSE, SUVmax, MTV and TLG using Cox proportional hazards regression. These parameters and bone metastasis were assessed separately for the univariate analysis and multivariate analysis for their ability to predict OS.

- The uptake in the bone marrow on 18F-FDG PET/CT images, as determined by the SUVmax and the different patterns of lesions between the positive bone marrow biopsy group and the negative group, were estimated using Mann-Whitney $\mathrm{U}$ tests and $\chi^{2}$ tests, respectively.

All tests were two-sided, and a probability of less than 0.05 was considered statistically significant.

\section{Results}

We retrospectively reviewed the records of sixty-eight paediatric patients (age $<18$ years) and finally 40 patients ( 31 at high-risk) were the subjects of this study. This study consisted of 21 females and 19 males with an average age of 37.78 months. All patients received standardized treatment according to their risk group. During the clinical follow-up period, $15(37.5 \%)$ patients experienced progression, among whom $9(22.5 \%)$ died. The characteristics of the patients are shown in Table 1.

Table 1. Comparison of clinical characteristics and pretreatment $\mathrm{PET} / \mathrm{CT}$ findings between progression and non-progression patients

\begin{tabular}{|c|c|c|c|c|}
\hline Characteristic & $\begin{array}{l}\text { Total } \\
(\mathrm{n}=40)\end{array}$ & $\begin{array}{l}\text { Non-progression } \\
(\mathrm{n}=25)\end{array}$ & Progression $(n=15)$ & $p$ \\
\hline Sex & 40 & & & 0.462 \\
\hline Female & & 12 & 9 & \\
\hline Male & & 13 & 6 & \\
\hline Age (month) & 40 & & 15 & 0.426 \\
\hline Mean $\pm S D$ (range) & $\begin{array}{l}37.78 \pm 22.958 \\
(6-84)\end{array}$ & $\begin{array}{l}35.400 \pm 21.670 \\
(6-80)\end{array}$ & $40.733 \pm 25.226(8-84)$ & \\
\hline Tumor location & 40 & & & 0.151 \\
\hline Retroperitoneal & & 21 & 10 & \\
\hline Mediastinal & & 4 & 3 & \\
\hline Other location & & 0 & 2 & \\
\hline Bone metastasis & 40 & & & 0.014 \\
\hline Yes & & 10 & 12 & \\
\hline No & & 15 & 3 & \\
\hline $\begin{array}{l}\text { Lymph node } \\
\text { metastasis }\end{array}$ & 40 & & & 0.123 \\
\hline Yes & & 14 & 12 & \\
\hline No & & 11 & 3 & \\
\hline $\begin{array}{l}\text { Other distant } \\
\text { metastases }^{\mathrm{a}}\end{array}$ & 40 & & & 0.182 \\
\hline Yes & & 8 & 8 & \\
\hline No & & 17 & 7 & \\
\hline BMI & 40 & & & 0.158 \\
\hline Yes & & 16 & 13 & \\
\hline No & & 9 & 2 & \\
\hline Risk stratification & 40 & & & 0.063 \\
\hline No-high risk & & 8 & 1 & \\
\hline high-risk & & 17 & 14 & \\
\hline MYCN amplification & $36^{\mathrm{a}}$ & & & 0.107 \\
\hline Yes & & 3 & 5 & \\
\hline No & & 20 & 8 & \\
\hline NSE (ug/L) & $39 \mathrm{~b}$ & 25 & 14 & 0.006 \\
\hline Median & 333.6 & 240.2 & 467.25 & \\
\hline Range & $22.81-1582$ & $22.81-1282$ & $120.8-1582$ & \\
\hline LDH (IU/L) & $39 \mathrm{~b}$ & 24 & 15 & 0.011 \\
\hline Median & 594 & 487.5 & 911 & \\
\hline Range & $88.2-5767$ & $88.2-2610$ & $430-5767$ & \\
\hline NSE (ug/L) & $39^{\mathrm{b}}$ & 25 & 14 & 0.006 \\
\hline Median & 333.6 & 240.2 & 467.25 & \\
\hline Range & $22.81-1582$ & $22.81-1282$ & $120.8-1582$ & \\
\hline SUVmax & 40 & 25 & 15 & 0.804 \\
\hline Median & 6.2 & 6.23 & 5.96 & \\
\hline Range & $2.63-57.03$ & $2.63-12.32$ & $3.14-57.03$ & \\
\hline TLG (g) & 40 & 25 & 15 & 0.004 \\
\hline Median & 325.43 & 227.25 & 626.97 & \\
\hline Range & $6.39-2009.28$ & $6.39-1179.97$ & $58.62-2009.28$ & \\
\hline $\operatorname{MTV}\left(\mathrm{cm}^{3}\right)$ & 40 & 25 & 15 & 0.001 \\
\hline Median & 92.11 & 82.44 & 189 & \\
\hline Range & $1.74-672$ & $1.74-192$ & $29.14-672$ & \\
\hline
\end{tabular}




\section{Predictive efficacy of NB between progression and non-progression}

In our study, there were significant differences in bone metastasis, NSE, LDH, MTV and TLG between patients with progression and those without; age, sex, MYCN amplification, GD2 expression, tumor location, lymph node metastasis, other distant metastasis, bone marrow involvement, risk stratification and SUVmax showed no significant differences between the two groups (Table 1).

\section{Survival analysis}

Age, LDH, NSE, bone metastasis, BMI, SUVmax, MTV and TLG were evaluated as variables in the survival analysis of patients with NB.

\section{Univariate and multivariate analysis for PFS}

In the univariate analysis of PFS, LDH, NSE, TLG, MTV and bone metastasis were determined to be statistically significant factors for PFS, although the effects of some of these factors were estimated to be relatively small (Table 2). In the multivariate analysis of PFS, MTV (Fig. 1A) and bone metastasis (Fig. 1B) were significant risk factors for PFS (Table 2).

Table 2. Univariate and multivariate analysis of prognostic factors for PFS

\begin{tabular}{|c|c|c|c|c|}
\hline \multirow[t]{2}{*}{ Variable } & \multicolumn{2}{|l|}{ Univariate analysis } & \multicolumn{2}{|l|}{ Multivariate analysis } \\
\hline & $\begin{array}{l}\text { HR } \\
\text { (95\% CI for HR) }\end{array}$ & $p$ & $\begin{array}{l}\text { HR } \\
(95 \% \text { CI for HR) }\end{array}$ & $p$ \\
\hline Age (month) & $0.998(0.976-1.019)$ & 0.827 & & \\
\hline LDH (IU/L) & $1.001(1.000-1.001)$ & 0.001 & & \\
\hline NSE (ug/L) & 1.001 (1.000-1.002) & 0.02 & & \\
\hline SUVmax & $1.024(0.983-1.065)$ & 0.254 & & \\
\hline TLG (g) & 1.001 (1.000-1.002) & 0.005 & & \\
\hline $\operatorname{MTV}\left(\mathrm{cm}^{3}\right)$ & 1.004 (1.002-1.007) & 0.002 & $6.820(2.093-22.223)$ & 0.001 \\
\hline Bone metastasis & 3.981 (1.094-14.49) & 0.036 & $4.841(2.106-18.759)$ & 0.023 \\
\hline BMI & $2.462(0.547-11.081)$ & 0.241 & & \\
\hline $\begin{array}{l}\text { Risk } \\
\text { classification }\end{array}$ & $4.323(0.565-33.073)$ & 0.158 & & \\
\hline
\end{tabular}

Univariate and multivariate analyses for OS

From the ROC analyses, the optimal cutoff values for LDH, NSE, SUVmax, MTV and TLG were $1064 \mathrm{IU} / \mathrm{L}, 364.4 \mathrm{IU} / \mathrm{L}, 12.01,191 \mathrm{~cm}^{3}$, and $341.41 \mathrm{~g}$, with AUCs of $0.7238,0.7410,0.4854,0.7148$ and 0.6661 , respectively. LDH, NSE and MTV showed significant differences in the univariate analysis of OS. In the multivariate analysis of OS, MTV (Fig. 1C) was the only significant risk factor for OS (Table 3). MTV was the only independent prognostic factor for both PFS and OS (Fig. 2) and other clinical characteristics or metabolic 18F-FDG PET/CT features were significantly associated with the survival outcomes for patients with NB (Tables $2 \& 3$ ).

Table 3. Univariate and multivariate analysis of prognostic factors for OS

\begin{tabular}{|c|c|c|c|c|c|c|}
\hline \multirow[t]{2}{*}{ Variable } & \multirow[t]{2}{*}{$\mathrm{n}$} & \multirow[t]{2}{*}{ Percent } & \multicolumn{2}{|l|}{ Univariate analysis } & \multicolumn{2}{|c|}{ Multivariate Analysis } \\
\hline & & & $\begin{array}{l}\mathrm{HR} \\
\text { (95\% CI for HR) }\end{array}$ & $\mathrm{P}$ & $\begin{array}{l}\text { HR } \\
\text { (95\% CI for HR) }\end{array}$ & $\mathrm{P}$ \\
\hline LDH (IU/L) & 39 & & $5.858(1.514-22.662)$ & 0.01 & & \\
\hline$\leq 1064$ & 29 & $74.4 \%$ & & & & \\
\hline$>1064$ & 10 & $25.6 \%$ & & & & \\
\hline NSE (ug/L) & 39 & & $5.143(1.064-24.867)$ & 0.042 & & \\
\hline$\leq 364.4$ & 22 & $56.41 \%$ & & & & \\
\hline$>364.4$ & 17 & $43.6 \%$ & & & & \\
\hline TLG(g) & 40 & & $2.560(0.639-10.259)$ & 0.184 & & \\
\hline$\leq 341.41$ & 21 & $52.5 \%$ & & & & \\
\hline$>341.41$ & 19 & $47.5 \%$ & & & & \\
\hline $\operatorname{MTV}\left(\mathrm{cm}^{3}\right)$ & 40 & & $8.221(2.048-32.996)$ & 0.003 & 7.772(1.939-31.159) & 0.004 \\
\hline$\leq 191$ & 31 & $77.5 \%$ & & & & \\
\hline$>191$ & 9 & $22.5 \%$ & & & & \\
\hline \multicolumn{3}{|c|}{ Bone metastasis } & $3.280(0.678-15.877)$ & 0.140 & & \\
\hline Yes & 22 & $55 \%$ & & & & \\
\hline No & 18 & $45 \%$ & & & & \\
\hline
\end{tabular}

\section{Correlation between semiquantitative PET/CT parameters and clinical features}

Lymph node metastasis analysis

Compared with patients without lymph node metastasis, patients with lymph node metastasis had higher TLG values $(\mathrm{p}=0.048<0.05)$.
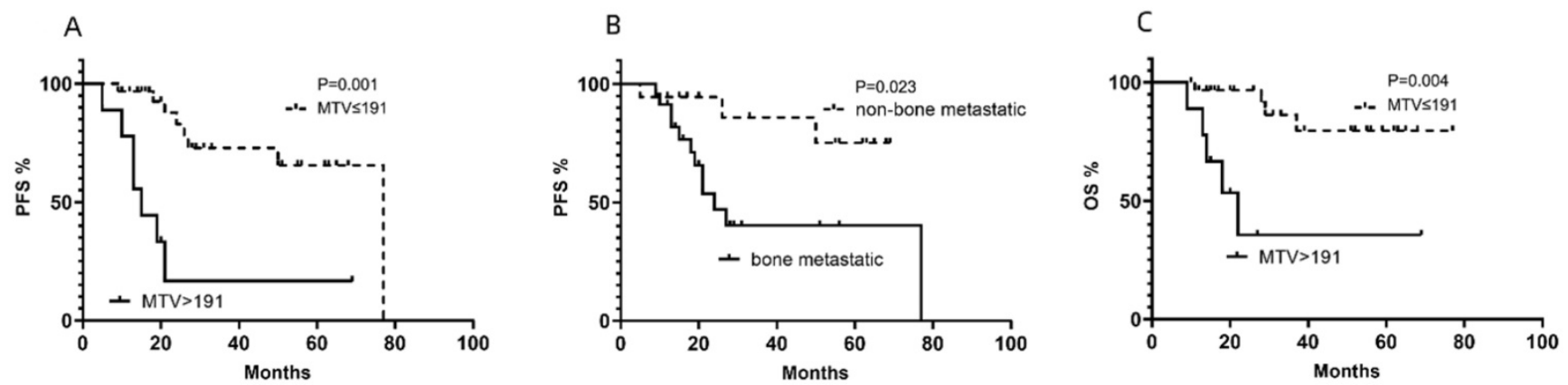

Figure 1. Cumulative progression-free survival (PFS) curves according to (A) metabolic tumor volume (MTV) and (B) bone metastasis and (C)cumulative overall survival (OS) curve according to MTV of NB lesions in enrolled patients. 

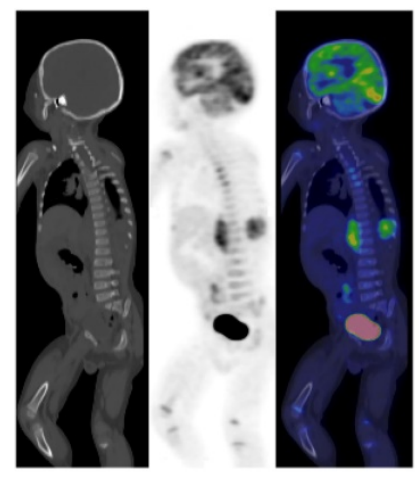

A
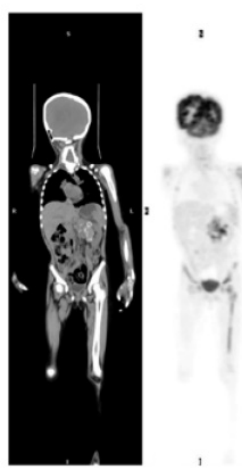
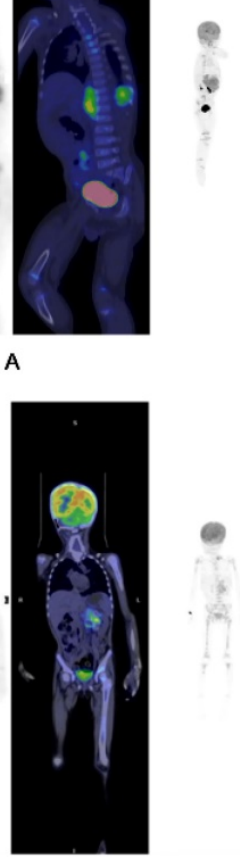

D

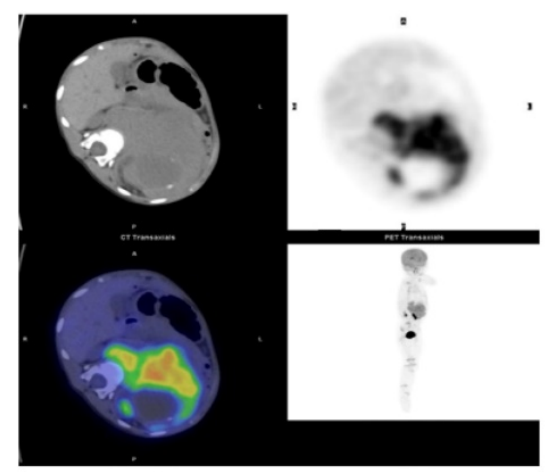

B

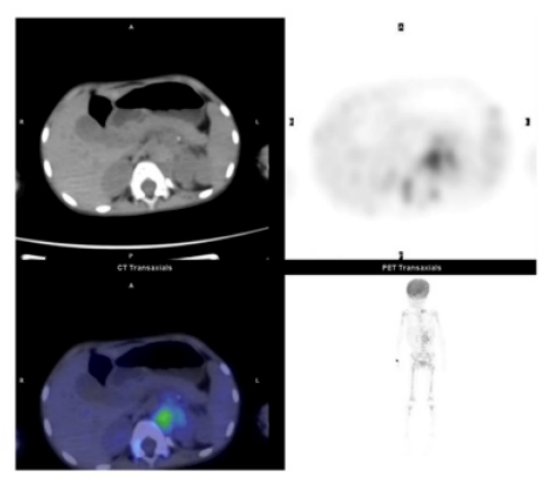

E
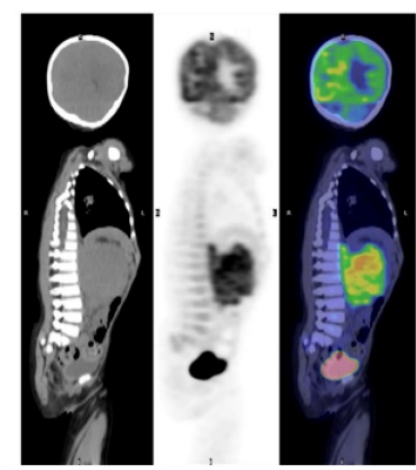

C
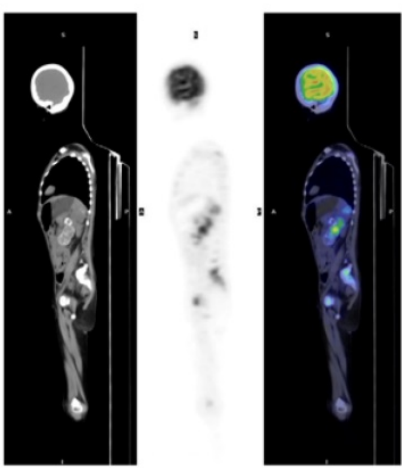

F

Figure 2. A-C) Representative images of a 1-year-old boy with stage IV NB. PET/CT revealed a primary tumor at the left suprarenal region, seen in the (A) coronal, (B) axial and (C) sagittal views. Intense metabolic tumor volume (MTV) (236) of the primary tumor was noted on the axial image. He progressed and eventually died. D-F) Representative images of a 4-year-old girl with stage IV NB. PET/CT revealed the primary tumor at left suprarenal region, seen in the (D) coronal, (E) axial, and (F) sagittal views. Small metabolic tumor volume (MTV) (46.9) of the primary tumor was noted on the axial image. She has not progressed yet.

Table 4. Spearman analysis between metabolic parameters of 18F-FDG PET/CT and serum biomarker levels

\begin{tabular}{lllll}
\hline Parameters & \multicolumn{2}{l}{ LDH $(\mathrm{IU} / \mathrm{L})$} & \multicolumn{2}{l}{ NSE $(\mathrm{ug} / \mathrm{L})$} \\
\cline { 2 - 5 } & $\mathrm{r}$ & $p$ & $\mathrm{r}$ & $p$ \\
\hline SUVmax & 0.23 & 0.153 & 0.321 & 0.044 \\
TLG $(\mathrm{g})$ & 0.51 & 0.001 & 0.440 & 0.005 \\
MTV $\left(\mathrm{cm}^{3}\right)$ & 0.456 & 0.003 & 0.357 & 0.024 \\
\hline
\end{tabular}

SUVmax, maximum standardized uptake values; TLG, total lesion glycolysis; MTV, metabolic tumor volume; $\mathrm{LDH}$, lactate dehydrogenase; NSE, neuron specific enolase.

\section{$\mathrm{BMI}$ analysis}

Before systemic therapy, unilateral or bilateral blind $\mathrm{BMB}$ of the posterior iliac crest were routinely performed in 40 patients, and the BMIs were verified in 29 patients. When analysing the PET/CT results of 40 patients, we recorded the bone marrow SUVmax of 40 patients, and their liver SUVmax and lesion-to-liver ratio (SUVmax-ratio) were calculated. We found that patients with positive BMBs had higher bone marrow SUVmax measurements than those with negative BMBs $(t=3.567, p \leq 0.001)$. From the ROC analyses, the optimal cutoff value for the SUVmax-ratio was 1.39, with an AUC of 0.703. The larger the SUVmax-ratio was, the worse the outcome $(z=2.123, p=0.034)$ (Fig. 3). In other words, the SUVmax-ratio can be further used to assess prognosis.

\section{COG risk classification}

Forty paediatric patients with NB were divided into a high-risk group and a non-high-risk group. Patients in the high-risk group had higher TLG than those in the non-risk group $(t=3.224, p=0.003)$.

\section{Serum biomarker levels}

Spearman analysis showed that the semiquantitative PET/CT parameters were positively correlated with the serum biomarkers levels (Fig. 4), except for SUVmax and LDH $(p=0.153)$ (Table 4). TLG, SUVmax, and MTV were compared separately based on the median of LDH (594 IU/L) and NSE (333.6 ug/L). The Mann-Whitney $U$ tests showed that compared with patients with low NSE levels, patients with high NSE levels had significantly higher TLG $(p=0.008)$. There was a tendency for correlations between NSE and MTV and SUVmax $(p=0.056$ and $p=0.072$, respectively). There was also a tendency for a correlation between the LDH level and TLG ( $p=0.068)$. However, no such correlation was found between the LDH level and MTV ( $\mathrm{p}=0.238)$.

\section{Discussion}

Evaluations to determine the disease stage in children with NB commonly includes imaging of the 
primary tumor site with CT or MRI to determine the primary tumor size and regional invasion and spread [1]. Although these methods can provide accurate anatomical information of the tumor, due to the metabolism of the tumor tissue and the surrounding tissue of the tumor, it is difficult and insufficient to accurately assess the true tumor volume with these methods. The current mainstream international functional imaging methods for NB include 18F-FDG PET/CT, I-123 MIBG and fluorine-18 fluorodeoxyphenylalanine (18F-DOPA) PET/CT. Unfortunately, I-123 MIBG and 18F-DOPA PET/CT are unavailable or less applicable in some countries. Because of the increased metabolism and accelerated proliferation, the tumor tissue consumes abundant glucose, which is manifested as increased FDG uptake [17]. Thus, the extent of FDG uptake can provide information about tumor disease activity. Previous studies have mostly focused on the diagnostic efficacy and significance of 18F-FDG PET/CT for staging [11, 12, 18-21]; however, to date, the prognostic value of this method is still not completely clear. Chao Li et al. [16] concluded that during subsequent treatment, patients with high focal bone marrow uptake and MTV and TLG on 18F-FDG PET/CT may have clearly inferior outcomes. In the present study, we enrolled 40 pediatric patients with newly diagnosed NB to evaluate the prognostic value 18F-FDG PET/CT.

Our results suggest that MTV, TLG, serum levels of LDH and NSE, and bone metastasis are important factors for survival. SUVmax, MTV and TLG have been recognized as prognostic factors in many malignancies [7, 21, 22]. The long-term follow-up analysis of the 40 patients in our study showed that the most discriminative MTV cut-off value had prognostic merit and MTV was a significant independent prognostic factor. MTV had a stronger association with both PFS and OS than other clinicopathological factors and metabolic parameters, which is similar to results of other studies [22, 23]. The results suggest that MTV may be an important factor during of planning treatment and the follow-up of NB patients.

In approximately $70 \%$ of patients, metastasis is present at the time of diagnosis and most commonly involves cortical bone and bone marrow [24]. BMI correlates with clinical stage and prognosis and is a frequent site of disease recurrence [25]. The detection of BMI also plays important role in predicting progression. In the present study, bone marrow SUVmax and the SUVmax-ratio were two meaningful parameters for NB patients with BMI. PET/CT has high sensitivity for assessing bone marrow infiltration in pediatric malignancies $[13,26]$. In particular, PET has improved sensitivity in detecting BMI in patients with lymphoma and may allow patients with positive BMI confirmed by PET/CT examinations to avoid undergoing invasive BMB [27, 28]. However, there is no clear conclusion on the significance of PET/CT for assessing the metabolism of tumor cells in the bone marrow of patients with NB. An important advantage of PET/CT over BMB is that PET/CT can assess all bone marrow sites at once and find unintended bone marrow infiltration in areas where biopsies are not usually performed [13]. The bone marrow manifests higher metabolic activity and FDG

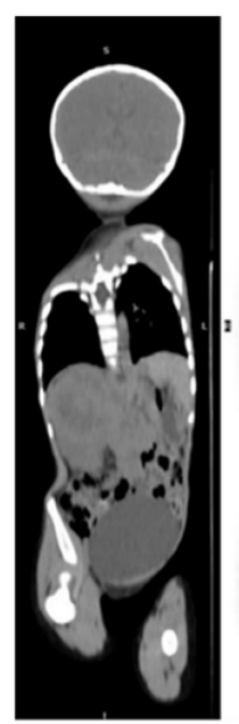

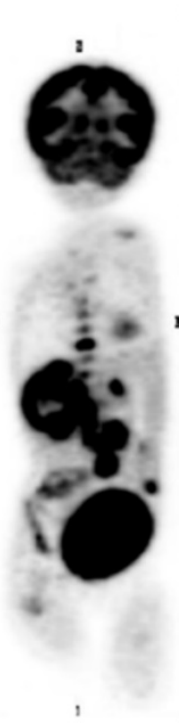

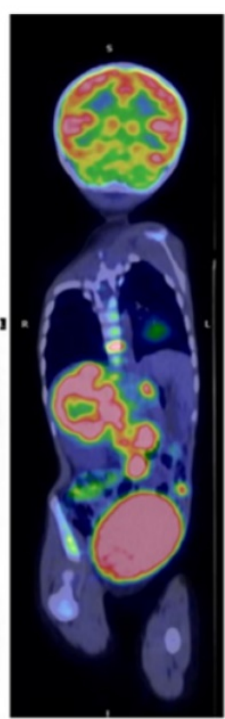

A

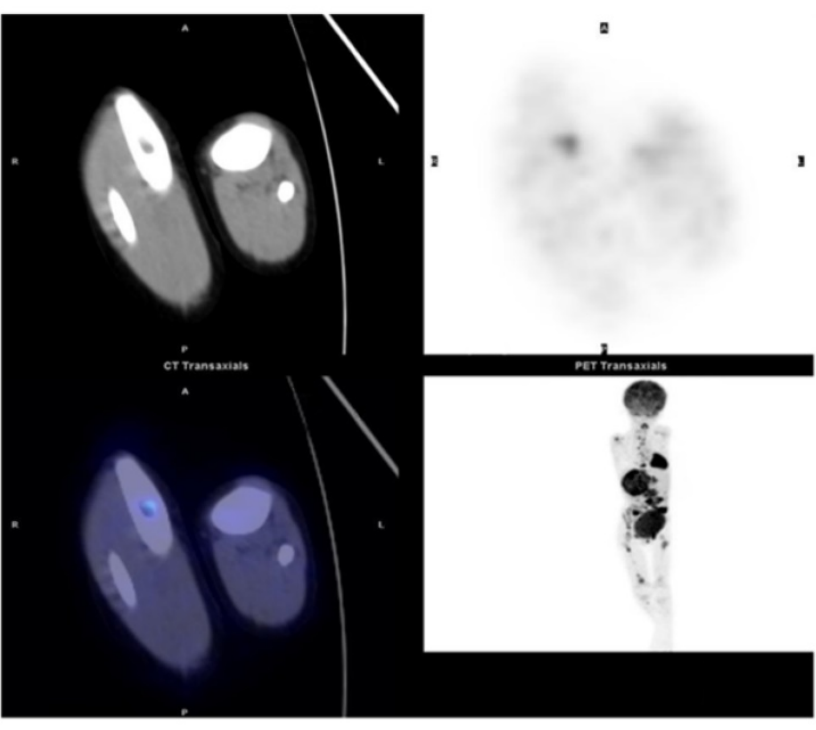

B

Figure 3. NB. Whole body PET/CT shows NB in the left adrenal gland. Bone marrow infiltration in the left and right femur head as well as the proximal tibia. (A) Activity in the right pelvis was observed. (B)Bone marrow infiltration in the left and right femurs. This patient had a large SUVmax-ratio value, 8.781 , and eventually the patient progressed, and eventually died, with very short progression-free survival (PFS) and overall survival (OS). 
uptake when tumor cells have transferred to the bone marrow, which can guide clinical diagnosis, staging and treatment. Because of its consistent results with $\mathrm{BMB}$ and its prognostic value in predicting progression, PET/CT has potential advantages as a non-invasive systemic examination. Our study demonstrated that the uptake intensity in the bone marrow may have significant prognostic implications and that 18F-FDG PET/CT has a substantial role in initially determining the BMI in $\mathrm{NB}$ patients. Additionally, the routine use of $\mathrm{BMB}$ of the posterior iliac crest may be reconsidered when 18F-FDG PET/CT is available. Considering the small number of cases, to some extent, this exploration was limited, and the bone marrow itself has physiological uptake and inflammation and several drugs can affect FDG PET/CT examinations. Therefore, whether 18F-FDG PET/CT can provide more information on BMI for patients with $\mathrm{NB}$, or even replace $\mathrm{BMB}$ still requires a larger number of clinical cases and more in-depth studies.

Serum LDH and NSE tests are simple, and these levels can be used as useful prognostic markers for NB [29-32]. However, serum LDH lacks the sensitivity and specificity to monitor disease activity. Serum NSE is more specific than LDH, but its sensitivity is still low. Regarding serum biomarkers, we demonstrated in this study that the serum LDH and NSE levels strongly correlated with TLG and MTV in 39 patients (the serum biomarker data of one of the forty patients were not available). This finding can be explained by
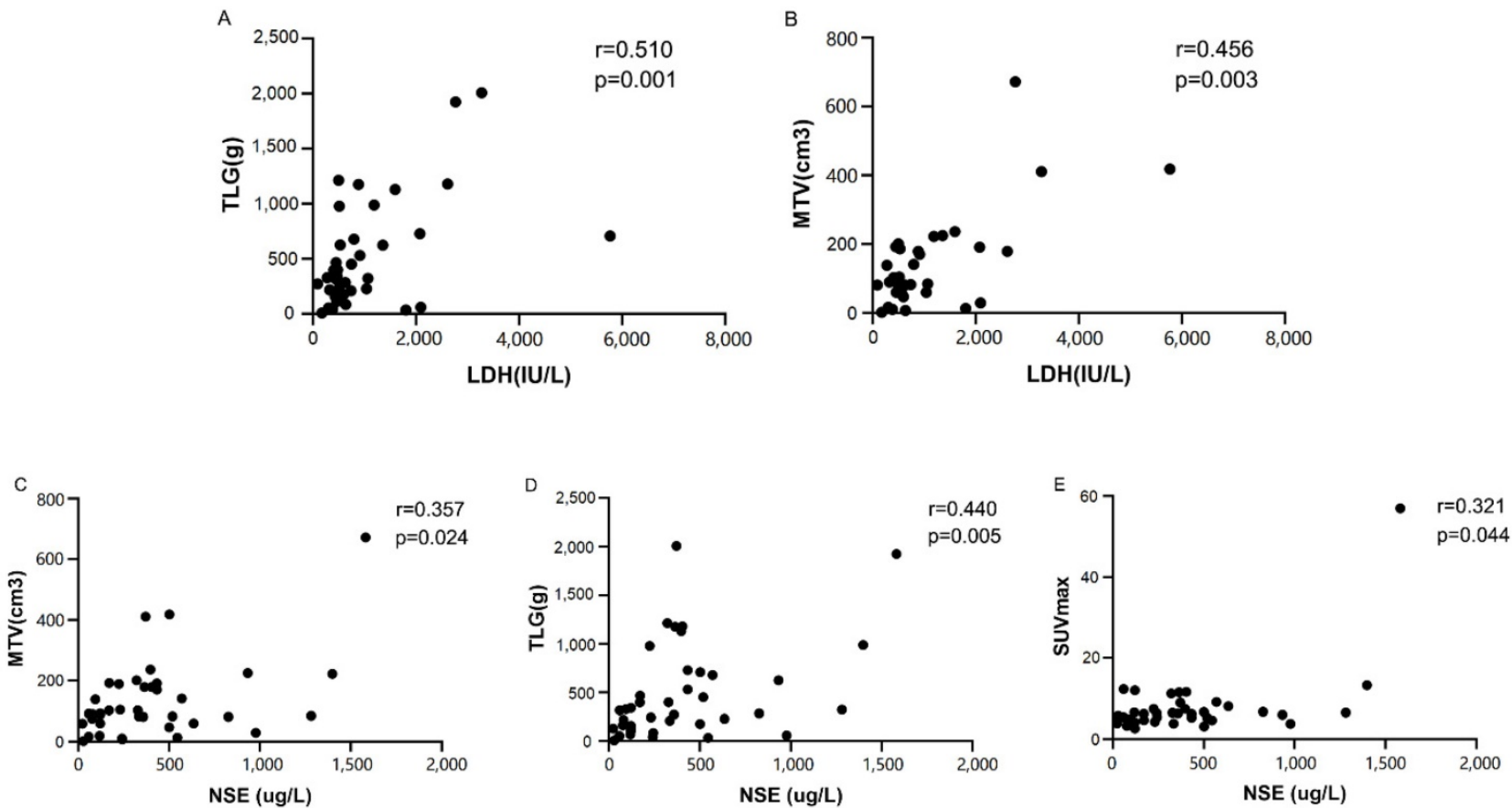

Figure 4. Spearman correlation analyses indicating a strong correlation between lactate dehydrogenase (LDH) and (A) total lesion glycolysis (TLG) or (B) metabolic tumor volume (MTV). Spearman correlation analyses indicating a strong correlation between the neuron-specific enolase (NSE) level and (C) metabolic tumor volume (MTV), (D) total lesion glycolysis (TLG) or (E) SUVmax 
Our research has several limitations. The statistical power was largely limited by the small number of subjects and the significance of the volumetric parameters might be more prominent with a larger sample size, considering the bulky and heterogeneous nature of NB. The tumor burden assessment based on the segmentation was performed only in primary tumor because the application of the technology to evaluate total tumor burden in children's solid tumors is difficult. On the other hand, NB tends to metastasize to the skull/skull base and the area is difficult to assess with FDG-PET due to the high FDG uptake in the brain. In some cases, a misestimation of the metabolic signal may interfere with the evaluation.

\section{Conclusions}

The metabolic parameters determined by pretreatment 18F-FDG PET/CT are a significant reflection of tumor burden and have predictive value for patients with NB. TLG and MTV, in particular, are very important. MTV and TLG are related to several clinical characteristics of NB, and they can express the tumor burden of NB; additionally, larger values of MTV and TLG may indicate disease progression. MTV is the only independent prognostic factor for both PFS and OS among SUVmax, TLG, and other prognostic factors. Patients with a higher MTV may have worse outcomes, including a shorter PFS and OS. Bone marrow SUVmax is also of great significance for the prognosis of NB. Pretherapeutic metabolic parameters determined by18F-FDG PET/CT may play important roles in the diagnosis and prognosis of paediatric patients with $\mathrm{NB}$, and large-scale prospective studies are also needed to validate the results by longer follow-up.

\section{Abbreviations}

18F-FDG: 2-deoxy-2-[18F] fluoro-D-glucose; PET/CT: positron emission tomography/computed tomography; NB: neuroblastoma; SUVmax: maximum standardized uptake value; MTV: metabolic tumor volume; TLG: total lesion glycolysis; PFS: progression-free survival; OS: overall survival; LDH: lactate dehydrogenase; NSE: neuron-specific enolase; COG: Children's Oncology Group; I-123 MIBG: iodine 123 metaiodobenzylguanidine; CR: complete remission; VGPR: very good partial response; BMI: bone marrow involvement; BMB: bone marrow biopsy; VOI: volume of interest; HL: Hodgkin's lymphoma; 18F-DOPA: fluorine-18 fluorodeoxyphenylalanine.

\section{Acknowledgements}

We acknowledge Tianjin Medical University
Cancer Institute and Hospital for support of samples and excellent conditions. She provides us with a good platform for this clinical research. This work was supported by the National Key R\&D Program of China under grant No. 2018YFC1313000.

\section{Funding}

This study was funded by the National Key R\&D Program of China (grant number 2018YFC1313000, 2018YFC1313001).

\section{Author Contributions}

Conceptualization, Shuai Man and Jie Yan; Data curation, Jianjing Liu; Formal analysis, Wenchao Ma; Funding acquisition, Qiang Zhao; Investigation, Shuai Man; Project administration, Jie Li; Resources, Jianjing Liu; Software, Shuai Man; Supervision, Qiang Zhao; Validation, Jie Yan, Jie Li and Yanna Cao; Visualization, Jianjing Liu; Writing - original draft, Shuai Man; Writing - review \& editing, Jie Yan.

\section{Competing Interests}

The authors have declared that no competing interest exists.

\section{References}

1. Whittle SB, Smith V, Doherty E, Zhao S, McCarty S, Zage PE. Overview and recent advances in the treatment of neuroblastoma. Expert review of anticancer therapy. 2017; 17: 369-86.

2. Sharp SE, Gelfand MJ, Shulkin BL. Pediatrics: diagnosis of neuroblastoma. Semin Nucl Med. 2011; 41: 345-53.

3. Pinto NR, Applebaum MA, Volchenboum SL, Matthay KK, London WB, Ambros PF, et al. Advances in Risk Classification and Treatment Strategies for Neuroblastoma. Journal of clinical oncology: official journal of the American Society of Clinical Oncology. 2015; 33: 3008-17.

4. Cohn SL, Pearson AD, London WB, Monclair T, Ambros PF, Brodeur GM, et al. The International Neuroblastoma Risk Group (INRG) classification system: an INRG Task Force report. Journal of clinical oncology: official journal of the American Society of Clinical Oncology. 2009· 27: 289-97.

5. Beyer T, Townsend DW, Brun T, Kinahan PE, Charron M, Roddy R, et al. A combined PET/CT scanner for clinical oncology. J Nucl Med. 2000; 41: 1369-79.

6. Cohade C, Wahl RL. Applications of positron emission tomography/ computed tomography image fusion in clinical positron emission tomography-clinical use, interpretation methods, diagnostic improvements. Semin Nucl Med. 2003; 33: 228-37.

7. Nakajima R, Matsuo Y, Kondo T, Abe K, Sakai S. Prognostic Value of Metabolic Tumor Volume and Total Lesion Glycolysis on Preoperative 18F-FDG PET/CT in Patients With Renal Cell Carcinoma. Clin Nucl Med. 2017; 42: e177-e82.

8. Kirchner J, Sawicki LM, Nensa F, Schaarschmidt BM, Reis H, Ingenwerth M, et al. Prospective comparison of (18)F-FDG PET/MRI and (18)F-FDG PET/CT for thoracic staging of non-small cell lung cancer. Eur J Nucl Med Mol Imaging. 2019; 46: 437-45

9. Ulaner GA, Castillo R, Wills J, Gonen M, Goldman DA. (18)F-FDG-PET/CT for systemic staging of patients with newly diagnosed ER-positive and HER2-positive breast cancer. Eur J Nucl Med Mol Imaging. 2017; 44: 1420-7.

10. Piccardo A, Lopci E, Conte M, Foppiani L, Garaventa A, Cabria M, et al. PET/CT imaging in neuroblastoma. The quarterly journal of nuclear medicine and molecular imaging : official publication of the Italian Association of Nuclear Medicine (AIMN) [and] the International Association of Radiopharmacology (IAR), [and] Section of the So. 2013; 57: 29-39.

11. Bleeker $\mathrm{G}$, Tytgat GA, Adam JA, Caron HN, Kremer LC, Hooft L, et al. 123I-MIBG scintigraphy and 18F-FDG-PET imaging for diagnosing neuroblastoma. The Cochrane database of systematic reviews. 2015: Cd009263.

12. Sharp SE, Shulkin BL, Gelfand MJ, Salisbury S, Furman WL. 123I-MIBG scintigraphy and 18F-FDG PET in neuroblastoma. J Nucl Med. 2009; 50: 1237-43

13. Zapata CP, Cuglievan B, Zapata CM, Olavarrieta R, Raskin S, Desai K, et al. $\mathrm{PET} / \mathrm{CT}$ versus bone marrow biopsy in the initial evaluation of bone marrow 
infiltration in various pediatric malignancies. Pediatric blood \& cancer. 2018; 65 .

14. Ishiguchi H, Ito S, Kato K, Sakurai $Y$, Kawai H, Fujita N, et al. Diagnostic performance of (18)F-FDG PET/CT and whole-body diffusion-weighted imaging with background body suppression (DWIBS) in detection of lymph node and bone metastases from pediatric neuroblastoma. Annals of nuclear medicine. 2018; 32: 348-62.

15. Liu CJ, Lu MY, Liu YL, Ko CL, Ko KY, Tzen KY, et al. Risk Stratification of Pediatric Patients With Neuroblastoma Using Volumetric Parameters of 18F-FDG and 18F-DOPA PET/CT. Clin Nucl Med. 2017; 42: e142-e8.

16. Li C, Zhang J, Chen S, Huang S, Wu S, Zhang L, et al. Prognostic value of metabolic indices and bone marrow uptake pattern on preoperative 18F-FDG PET/CT in pediatric patients with neuroblastoma. Eur J Nucl Med Mol Imaging. 2018; 45: 306-15.

17. Nakatsuka Y, Handa T, Nakamoto Y, Nobashi T, Yoshihuji H, Tanizawa K, et al. Total lesion glycolysis as an IgG4-related disease activity marker. Modern rheumatology. 2015; 25: 579-84.

18. Xia J, Zhang H, Hu Q, Liu SY, Zhang LQ, Zhang A, et al. Comparison of diagnosing and staging accuracy of PET (CT) and MIBG on patients with neuroblastoma: Systemic review and meta-analysis. Journal of Huazhong University of Science and Technology Medical sciences $=$ Hua zhong ke ji da xue xue bao Yi xue Ying De wen ban = Huazhong keji daxue xuebao Yixue Yingdewen ban. 2017; 37: 649-60.

19. Dhull VS, Sharma P, Patel C, Kundu P, Agarwala S, Bakhshi S, et al. Diagnostic value of 18F-FDG PET/CT in paediatric neuroblastoma: comparison with 131I-MIBG scintigraphy. Nuclear medicine communications. 2015; 36: 1007-13.

20. Mueller WP, Coppenrath E, Pfluger T. Nuclear medicine and multimodality imaging of pediatric neuroblastoma. Pediatr Radiol. 2013; 43: 418-27.

21. Kiratli PO, Tuncel M, Bar-Sever Z. Nuclear Medicine in Pediatric and Adolescent Tumors. Semin Nucl Med. 2016; 46: 308-23.

22. Hofheinz F, Li Y, Steffen IG, Lin Q, Lili C, Hua W, et al. Confirmation of the prognostic value of pretherapeutic tumor SUR and MTV in patients with esophageal squamous cell carcinoma. Eur J Nucl Med Mol Imaging. 2019; 46: 1485-94.

23. Akagunduz OO, Savas R, Yalman D, Kocacelebi K, Esassolak M. Can adaptive threshold-based metabolic tumor volume (MTV) and lean body mass corrected standard uptake value (SUL) predict prognosis in head and neck cancer patients treated with definitive radiotherapy/chemoradiotherapy? Nuclear medicine and biology. 2015; 42: 899-904.

24. Choi YJ, Hwang HS, Kim HJ, Jeong YH, Cho A, Lee JH, et al. (18)F-FDG PET as a single imaging modality in pediatric neuroblastoma: comparison with abdomen CT and bone scintigraphy. Annals of nuclear medicine. 2014; 28: 304-13.

25. Burchill SA, Beiske K, Shimada H, Ambros PF, Seeger R, Tytgat GA, et al. Recommendations for the standardization of bone marrow disease assessment and reporting in children with neuroblastoma on behalf of the International Neuroblastoma Response Criteria Bone Marrow Working Group. Cancer. 2017; 123: 1095-105.

26. Yagci-Kupeli B, Kocyigit-Deveci E, Adamhasan F, Kupeli S. The Value of 18F-FDG PET/CT in Detecting Bone Marrow Involvement in Childhood Cancers. J Pediatr Hematol Oncol. 2019; 41: 438-41.

27. Agrawal K, Mittal BR, Bansal D, Varma N, Srinivasan R, Trehan A, et al. Role of F-18 FDG PET/CT in assessing bone marrow involvement in pediatric Hodgkin's lymphoma. Annals of nuclear medicine. 2013; 27: 146-51.

28. Cheng G, Chen W, Chamroonrat W, Torigian DA, Zhuang H, Alavi A. Biopsy versus FDG PET/CT in the initial evaluation of bone marrow involvement in pediatric lymphoma patients. Eur J Nucl Med Mol Imaging. 2011; 38: 1469-76.

29. Berthold F, Engelhardt-Fahrner U, Schneider A, Schumacher R, Zieschang J. Age dependence and prognostic impact of neuron specific enolase (NSE) in children with neuroblastoma. In vivo (Athens, Greece). 1991; 5: 245-7.

30. Isgro MA, Bottoni P, Scatena R. Neuron-Specific Enolase as a Biomarker: Biochemical and Clinical Aspects. Advances in experimental medicine and biology. 2015; 867: 125-43.

31. Brodeur GM, Pritchard J, Berthold F, Carlsen NL, Castel V, Castelberry RP, et al. Revisions of the international criteria for neuroblastoma diagnosis, staging, and response to treatment. Journal of clinical oncology : official journal of the American Society of Clinical Oncology. 1993; 11: 1466-77.

32. Simon T, Hero B, Hunneman DH, Berthold F. Tumour markers are poor predictors for relapse or progression in neuroblastoma. European journal of cancer (Oxford, England: 1990). 2003; 39: 1899-903.

33. Jurisic V, Radenkovic S, Konjevic G. The Actual Role of LDH as Tumor Marker, Biochemical and Clinical Aspects. Advances in experimental medicine and biology. 2015; 867: 115-24.

34. Albano D, Bertoli M, Battistotti M, Rodella C, Statuto M, Giubbini R, et al Prognostic role of pretreatment 18F-FDG PET/CT in primary brain lymphoma. Annals of nuclear medicine. 2018; 32: 532-41.

35. Alavi A, Werner TJ, Hoilund-Carlsen PF, Zaidi H. Correction for Partial Volume Effect Is a Must, Not a Luxury, to Fully Exploit the Potential of Quantitative PET Imaging in Clinical Oncology. Molecular imaging and biology. 2018; 20: 1-3.

36. London WB, Bagatell R, Weigel BJ, Fox E, Guo D, Van Ryn C, et al. Historical time to disease progression and progression-free survival in patients with recurrent/refractory neuroblastoma treated in the modern era on Children's Oncology Group early-phase trials. Cancer. 2017; 123: 4914-23. 